\title{
Postoperative Complications, Patient Safety, and Comfort of the Oral Fiberoptic Bronchoscopy-guided Intubation Compared with the Oral laryngoscope Intubation
}

\author{
Troels Edsen ${ }^{1, ~ *, ~ M a r i a n ~ P e t e r s e n ², ~ F i n n ~ B o r g b j e r g ~ M o l t k e ~}{ }^{1,3}$, Carrinna Aviaja Hansen ${ }^{1}$ \\ ${ }^{1}$ Department of Neuroanesthesiology, the Neuroscience Centre, Rigshospitalet, University of Copenhagen, Copenhagen, Denmark \\ ${ }^{2}$ Neuroscience Center, Rigshospitalet, University of Copenhagen, Copenhagen, Denmark \\ ${ }^{3}$ Department of Veterinary Clinical and Animal Science, University of Copenhagen, Copenhagen, Denmark
}

Email address:

troels.edsen@regionh.dk (T. Edsen)

*Corresponding author

\section{To cite this article:}

Troels Edsen, Marian Petersen, Finn Borgbjerg Moltke, Carrinna Aviaja Hansen. Postoperative Complications, Patient Safety, and Comfort of the Oral Fiberoptic Bronchoscopy-guided Intubation Compared with the Oral laryngoscope Intubation. Science Journal of Public Health. Vol. 8, No. 4, 2020, pp. 99-107. doi: 10.11648/j.sjph.20200804.12

Received: May 5, 2020; Accepted: June 15, 2020; Published: June 29, 2020

\begin{abstract}
Introduction: Postoperative sore throat (POST) and hoarseness is an on-going worldwide challenge after general anaesthesia. In this study, we hypothesised that patients intubated with the fiberoptic bronchoscope-guided intubation device differs in POST and hoarseness from other patients intubated with the oral Macintosh laryngoscope-guided intubation device. If this could be confirmed, the guideline of peri anaesthesia may need to be different. Objective: This study aimed to investigate whether fiberoptic intubation can provide increased patient safety and comfort compared to standard laryngoscope intubation performed by nurse anaesthetists. Methods: Within this single-blinded, consecutively controlled clinical trial two hundred and nine patients scheduled for elective neurosurgical treatment requiring intratracheal intubation, were randomly divided into intervention and control group. The intervention group received fiberoptic bronchoscopy-guided intubation during anaesthesia as well as nursing and postoperative treatment based on conventional drug therapy after neurosurgical treatment. The control group received intubation during anaesthesia, as usual, using Macintosh laryngoscope intubation, and postoperative treatment based on conventional drug therapy after neurosurgical treatment. Data regarding the patient safety were obtained during peri anaesthesia, and data concerning the patient comfort were collected on the first postoperative day. Statistical analyses were performed using SPSS version 22. Results: Seven patients were excluded (3.3\%); thus, the final population consisted of two hundred and two patients: $62.4 \%$ women, the mean age of 52 years (20 to 86 years), 106 (52.5\%) patients in the fiberoptic intubation group. A statistically significant difference was found between groups according to patient safety, measured by the number of anaesthetists involved $\mathrm{P}=0.024$ in favour of the fiberoptic intubation device. A trend was pointing to increased pain in the mouth and throat $\mathrm{P}=0.053$, when intubated with the laryngoscope. Other patient safety and comfort measurements showed no statistically significant differences between groups. Conclusions: The study directs attention to some possible benefits of using the fiberoptic intubation device, which may improve patient safety and comfort when compared to standard laryngoscope intubation.
\end{abstract}

Keywords: Sore Throat, Perioperative Complications, Endotracheal Intubation, Hoarseness, Postoperative Sore Throat

\section{Introduction}

Postoperative sore throat (POST) and hoarseness are minor but common and frequent complaints after general anaesthesia; these complications are known to affect patients satisfaction and recovery negatively in patients undergoing endotracheal intubation [1]. Studies investigating POST has reported POST in 30-66\% of surgical patients undergoing general anaesthesia [2-5]. Several and very different treatments have been investigated to reduce the occurrence of this complication, such as tests with tube cuff shape and thermal softening $[6,7]$. Different usage of a variety of drugs 
has been tested, e.g. lidocaine [8], remifentanil [9], steroids [10] and NSAID [11-13] used intravenously, topically as well as gargling or spay $[14,15]$. However, El-Boghdadly and colleagues found in a systematic review that no single intervention is quite useful by themselves [1]. The comprehensive effort over the years to try to minimise POST and hoarseness is a testify of a great need for improving postoperative patient satisfaction, as well as a need to improve the patient comfort, since these complications may affect the individuals' activity level after discharge from hospital $[16,17]$. Thus, it is crucial to continue to seek ways of minimising these postoperative adverse events. The sources of POST and hoarseness is multifaceted [2, 17, 18]; regardless, the tissue damage of the throat and upper airway may be considered as an overriding causative factor. Thus, it is required to use an intubation device that will provide the most secure and gentle intubation session. The primarily used device for intubation in the current department and many other departments is the Macintosh laryngoscope, which is a well-documented source of POST and hoarseness [2]. This, leading to relevant considerations of the appropriateness of testing, evaluating and possibly implementing other intubation devices.

Intubation with the fiberoptic bronchoscope-guided intubation device can be performed without stimulation of oropharyngeal structures [19], the benefits of this method have been found in a study investigating nasotracheal intubation [3]. The nasotracheal intubation, as a general procedure in neurosurgery, is not appropriate because a nasal tube will be causing a risk of damaging the pituitary structures in the upper nose areas, different from the conventional orotracheal intubation. Therefore, oral fiberoptic bronchoscope-guided intubation is relevant to be tested in speak of neurosurgical procedures; it is hypothesised to have the potential to reduce POST and hoarseness compared to the use of the oral Macintosh laryngoscope. However, no studies were found to comparing the oral fiberoptic bronchoscope-guided intubation and the oral Macintosh laryngoscope intubation, measured by the incidence of POST and hoarseness, the time of secured airway, duration of intubation, number of intubation-attempts and number of nurse anaesthetist involved until secured airway.

Our hypothesis is, that patients intubated with the fiberoptic bronchoscope-guided intubation device differ in POST and hoarseness from other patients intubated with the oral Macintosh laryngoscope-guided intubation device. If this could be confirmed, the guideline of peri anaesthesia may need to be different.

The study aims to evaluate the occurrence of postoperative complications and possible advantages of using the fiberoptic bronchoscope-guided device versus the Macintosh laryngoscope-guided intubation device. Secondly, to investigate patient safety during the intubation session. The study results will provide a noteworthy basis of clinical guidelines.

\section{Method}

\subsection{Design}

The current study is a single-blinded, consecutively controlled clinical trial. It was impossible to blind the nurse anaesthetist according to the intubation procedure type, leading to a single-blinded study. None of the patients was interviewed by the nurse anaesthetist, who carried out the intubation procedure.

\subsection{Definitions}

Patient safety is a broad term. In this article, it is used as a term for an intubated secured airway, and prevention of tissue damage. Patient safety is measured as: time used until secured airway, the duration of intubation time, the number of intubation-attempts until secured airway, and the number of nurse anaesthetists involved in the completion of intubation.

The time to complete the intubation was a measurement of minutes. The time began when the anaesthesia was initiated and until the airway was secured.

The duration of intubation time was a measurement of minutes, beginning when the anaesthesia was initiated and until the anaesthesia was completed and the patient extubated.

The number of intubation attempts was a measurement of how many times, the nurse anaesthetist attempted to intubate until secured airway was established.

The number of anaesthetists involved was a measurement of the number of nurse anaesthetists, who made an intubation attempt on the same patient until secured airway was achieved.

Sore throat and pain was measured on the first day after the surgery. The visual analogue scale (VAS 0-10) was used as the measuring instrument.

Hoarseness was measured on the first day after the surgery. No validated scale for measuring hoarseness was found. Therefore, in this study; we used a simple 4-point scale measuring the experience of hoarseness by none, slight, moderate, or pronounced hoarseness.

The data of sore throat, pain and hoarseness were selfreported pre-surgery and the day after the surgery.

\subsection{Power Calculation}

A power calculation was conducted, based on pain in the throat/mouth (the primary outcome) scored on a VAS. Biro et al. [2] measured the mean level of pain in the throat on the first postoperative day VAS $=28$ on a 100 -point Likert scale. Based on this, with a significance level of $95 \%(\alpha=5)$ and strength of $80 \%(\beta=20)$, a variance of 18.76 [2], with a required difference between the control group and the intervention group on $20 \%$, it was estimated that 88 patients should be included in each group. The risk of dropouts was estimated at $10 \%$, thus allocating at least 97 patients in each group. 


\subsection{The Data Collection Group and Skills}

Three nurse anaesthetists were responsible for collecting data: the first author, the senior nurse of the department, and a third nurse anaesthetist.

The entire staff of nurse anaesthetists (14 in total) in the department of the Neuroanesthesiology unit performed the intubations included in this study. Their experience in laryngoscopic intubations was based on several years of performing such procedures. While the experience of fiberoptic intubation was acquired from a skills training course carried out immediately before this study [19]. The nurse anaesthetists had achieved the experience of at least 20 independent fiberoptic intubations, before participating in the study.

In Denmark, nurse anaesthetists hold a specialised education in anesthesiological nursing, which is a 2-year education position. In beforehand, Danish authorisation as a registered nurse (known as a certified registered nurse in the United States) is required, together with at least two years of clinical nursing experience. In the Danish healthcare system, the nurse anaesthetists perform the anaesthetic management to a considerable extent in collaboration with the anesthesiologist.

\subsection{Sampling}

Inclusion criteria based on the pre-anesthesiological assessment: Adult patients aged $\geq 18$ years suitable for both intubation methods tested in the study. Patients requiring general anaesthesia for an elective neurosurgical intervention. Patient without known airway difficulties. Patients with normal cognitive functions.

Exclusion criteria: Patients scheduled for surgery on the upper cervical spine (due to the guideline for this type of surgery requiring fiberoptic intubation). Surgical procedures which require nasal intubation. Patients with a known hiatus hernia. Patients with reflux. Patients requiring intensive care treatment postoperatively.

\subsection{Randomisation}

The randomisation process was a so-called "draw-up procedure" having sealed envelopes with descriptions of the type of intubation procedure. There were 120 numbered envelopes for each of the two groups, 1) the oral fiberoptic bronchoscope-guided intubation and 2) oral Macintosh laryngoscope intubation.

Expected possible dropouts, would be the withdrawal of patient consent for participation on the day of surgery; the need for changing the intubation procedure due to an acute situation; lack of data registered during the procedure for the current study; or patient not fasting according to the guidelines. Patients, that met the inclusion criteria, were randomly assigned to one of the two groups. The 240 envelopes were shuffled, and a nurse from the intervention group drew the envelope. Inclusion ceased at the point when the number of patients for both groups was reached, based on the power calculation. Patients were included during January 2011 and October 2012. The envelope followed the patient, from the pre-anaesthesia evaluation and was opened by the nurse anaesthetist during preparation, immediately before the surgical procedure. The pre-anaesthesia evaluation included the Mallampati score, receding chin registration, possible underbite, neck range of motion, and earlier Cormac-Lehane classification was carried out by the anesthesiologist.

\subsection{Ethical Considerations}

Individuals suitable for participation received both oral and written information on the purpose of the study. If the patient agreed to participate in the study, the participation form was filled out, and the patient was assigned to randomisation by the data collection group. The study followed the ethical principles of the World Medical Association Declaration of Helsinki [20]. All participating patients had the opportunity to withdraw their permission to participate, without any consequences for their treatment.

The study consisted of two accepted intubation methods, and the patients have not been exposed to any experimental intervention. The local medical ethics committee at Rigshospitalet, University of Copenhagen, Denmark approved the study j.nr 2007-58-0015, as did The Danish Data Protection Agency, j.nr. 30-0494, following the directives for data retention.

The database generated during the current study is not publicly available due to the policy of the Danish Data Protection Agency. The data are considered confidential. Anonymised data files are available from the corresponding author on reasonable request.

\subsection{Data Collection}

The nurse anaesthetists filled out the usual registration before surgery, as well as the study datasheet, which included the following measuring points:

1. Intubation time - the time in minutes until the secured airway

2. Duration of intubation time in minutes

3. The number of anaesthetists involved in the intubation until secured airway was achieved

4. The number of attempts until the secured airway

5. Intubation misconduct; defined as the tube in the oesophagus

6. The evaluation of the throat was defined based on the Cormack-Lehane scale. In both the fiberoptic intubation and the laryngoscopic situations

7. Unexpected changes in the intubation procedure were recorded

The day after the surgery, a member of the data collection group interviewed the patient following a structured questionnaire, containing the questions addressing the patient's self-reported experience of pain in the throat, mouth and hoarseness, compared to before surgery; as well as checking for any dental damage occurred during surgery. The 
pain was measured using the VAS range $0-10$, understanding 0 as no pain at all, and ten as the worst imaginable pain. Hoarseness was measured using a simple 4-point scale (none, light, moderate, pronounced). Patients were blinded concerning the intubation method, although on request, the patients were informed, after the postoperative data collection.

The patients were anaesthetised with Propofol and Ultiva, in a total intravenous anaesthesia setup (TIVA). According to procedure guidelines, the dosage of Propofol was set at $0.5 \mathrm{ml} / \mathrm{kg} /$ hour, and induction dosage was 2 $\mathrm{mg} / \mathrm{kg}$. The Ultiva induction dosage was 1 mikrogram $/ \mathrm{kg} / \mathrm{min}$, and the steady-state dosage was 0.5 mikrogram $/ \mathrm{kg} / \mathrm{min}$. The patients also received cisatracurium as a neuromuscular blocking agent after the administration of the anaesthesia in a dosage of $0.1 \mathrm{mg} / \mathrm{kg}$. Following the surgery, all the patients received the same postoperative analgesic treatment immediately after anaesthesia of $0,1 \mathrm{mg} / \mathrm{kg}$ morphine, and to prevent nausea, $4 \mathrm{mg}$ of Ondansetron.

\section{Analyses}

Statistical analyses were performed using SPSS (version 22 for Windows). Descriptive statistics were used to describe the baseline patient characteristics (Table 1), type of intubation (Table 2), and pain and a sore throat (Table 3 ). A linear logistic regression analysis was conducted, to compare the oral fiberoptic bronchoscope-guided intubation versus oral Macintosh laryngoscope intubation, including chosen covariates (Table 4). The covariates selected for entry into the model of the final linear logistic regression analysis were chosen, based on a forward pairwise linear regression analysis with criteria used for the probability of $F$ pin (0.98) and pout (0.99). A model of six variables was chosen based on the adjusted R Square 0.087 (decreasing after model 6) and statistically significant $\mathrm{F}$ Change, $F(1.19)=1.645, P=0.20$. Thus, the linear logistic regression analysis used pairwise analysis with a critical $\mathrm{P}<0.20$.

Table 1. Baseline.

\begin{tabular}{|c|c|c|c|c|}
\hline & $\begin{array}{l}\text { Male } \mathrm{N}=76(37.6 \%) \mathrm{n}(\% \\
\text { within gender/\%total })\end{array}$ & $\begin{array}{l}\text { Female } \mathrm{N}=126(62.4 \%) \mathrm{n} \\
(\% \text { within gender/total) }\end{array}$ & $\mathbf{P}$ & $\begin{array}{l}\text { Total } N=202 n \\
(\% \text { total) }\end{array}$ \\
\hline \multicolumn{5}{|l|}{ Age at treatment } \\
\hline \multicolumn{5}{|l|}{ Mean 52 / Median 53 years } \\
\hline 1st quartile ( $18-42$ years) $n=53$ & $23(30.3 / 11.4)$ & $30(23.8 / 14.6)$ & 0.314 & $53(26.2)$ \\
\hline 2nd quartile ( $43-53$ years) $n=53$ & $20(26.3 / 9.9)$ & $33(26.2 / 16.3)$ & 0.984 & $53(26.2)$ \\
\hline 3 rd quartile (54-64 years) $n=50$ & $17(22.3 / 8.4)$ & $33(26.2 / 16.3)$ & 0.543 & $50(24.8)$ \\
\hline 4 th quartile ( $65-86$ years) $n=46$ & $16(21.1 / 7.9)$ & $30(23.8 / 14.9)$ & 0.652 & $46(22.8)$ \\
\hline \multicolumn{5}{|l|}{ Intubation type } \\
\hline $\begin{array}{l}\text { oral fiberoptic bronchoscope-guided device } \\
\text { (intervention) }\end{array}$ & $50(65.8 / 24.8)$ & $56(44.4 / 27.7)$ & 0.003 & $106(52.5)$ \\
\hline oral Macintosh laryngoscope-guided device (usual) & $26(34.2 / 12.9)$ & $70(55.6 / 34.7)$ & 0.003 & $96(47.5)$ \\
\hline \multicolumn{5}{|l|}{ Pain } \\
\hline Mouth non or light (VAS $0-2$ ) & $72(94.7 / 35.6)$ & $121(96.0 / 59.9)$ & 0.666 & $193(95.5)$ \\
\hline Mouth medium (VAS 3-4) & $0(0.0 / 0.0)$ & $2(1.6 / 1.0)$ & 0.271 & $2(1.0)$ \\
\hline Mouth medium-severe (VAS 5-6) & $1(1.3 / 0.5)$ & $2(1.6 / 1.0)$ & 0.877 & $3(1.5)$ \\
\hline Mouth severe (VAS 7-8) & $3(4.0 / 1.5)$ & $1(0.8 / 0.5)$ & 0.120 & $4(2.0)$ \\
\hline Throat non or light (VAS $0-2$ ) & $71(93.4 / 35.2)$ & $119(94.4 / 58.9)$ & 0.766 & $190(94.1)$ \\
\hline Throat medium (VAS 3-4) & $3(4.0 / 1.5)$ & $6(4.8 / 3.0)$ & 0.786 & $9(4.5)$ \\
\hline Throat Mouth medium-severe (VAS 5-6) & $2(2.6 / 1.0)$ & $1(0.8 / 0.5)$ & 0.297 & $3(1.5)$ \\
\hline \multicolumn{5}{|l|}{ Hoarseness } \\
\hline Non (VAS 0) & $56(73.7 / 27.7)$ & $87(69.1 / 43.1)$ & 0.484 & $143(70.8)$ \\
\hline Light (VAS 1-2) & $19(25.0 / 9.4)$ & $38(30.2 / 18.8)$ & 0.431 & $57(28.2)$ \\
\hline Moderate (VAS 3-4) & $1(1.3 / 0.5)$ & $1(0.8 / 0.5)$ & 0.717 & $2(1.0)$ \\
\hline \multicolumn{5}{|l|}{ BMI } \\
\hline Underweight $(<18.5)$ & $1(1.3 / 0.5)$ & $2(1.6 / 1.0)$ & 0.877 & $3(1.5)$ \\
\hline Normal weight $(18.5-24.9)$ & $28(36.8 / 13.7)$ & $62(49.2 / 30.7)$ & 0.088 & $90(44.6)$ \\
\hline Overweight (25-29.9) & $38(50.0 / 18.8)$ & $41(32.5 / 20.3)$ & 0.014 & $79(39.1)$ \\
\hline Severe overweight $(\geq 30)$ & $9(11.8 / 4.5)$ & $20(15.9 / 9.9)$ & 0.430 & $29(14.4)$ \\
\hline \multicolumn{5}{|l|}{ Number of intubation attempt } \\
\hline Normal (1-2) & $73(96.1 / 36.1)$ & $122(96.8 / 60.4)$ & 0.772 & $195(96.5)$ \\
\hline Over normal $(\geq 3)$ & $3(4.0 / 1.5)$ & $2(1.6 / 1.0)$ & 0.297 & $5(2.5)$ \\
\hline \multicolumn{5}{|l|}{ Duration of intubation, time } \\
\hline 1st quartile (58-142 minutes) & $22(29.0 / 10.9)$ & $31(24.6 / 15.4)$ & 0.498 & $53(26.2)$ \\
\hline 2nd quartile (143-175 minutes) & $23(30.3 / 11.4)$ & $26(20.6 / 12.9)$ & 0.123 & $49(24.3)$ \\
\hline 3rd quartile (176-220 minutes) & $17(22.4 / 8.4)$ & $36(28.6 / 17.8)$ & 0.333 & $53(26.2)$ \\
\hline 4 th quartile (221-442 minutes) & $14(18.4 / 6.9)$ & $33(26.2)$ & 0.207 & $47(23.3)$ \\
\hline \multicolumn{5}{|l|}{ Number of anaesthetist involved } \\
\hline 1 & $73(96.1 / 36.1)$ & $115(91.3 / 59.9)$ & 0.196 & $188(93.1)$ \\
\hline 2 & $3(4.0 / 1.5)$ & $9(7.1 / 4.5)$ & 0.353 & $12(5.9)$ \\
\hline
\end{tabular}


Table 2. Intubation type.

\begin{tabular}{|c|c|c|c|}
\hline & $\begin{array}{l}\text { Fiberoptic } \mathrm{N}=10652.5 \%) n \text { (\% within } \\
\text { intubation type/\% total) }\end{array}$ & $\begin{array}{l}\text { Laryngoscope } \mathrm{N}=96(47.5 \%) n(\% \\
\text { within intubation type } / \% \text { total) }\end{array}$ & $\mathbf{P}$ \\
\hline \multicolumn{4}{|l|}{ Gender } \\
\hline Male & $50(47,2 / 24,8)$ & $26(27,1 / 12,9)$ & $0.003^{* *}$ \\
\hline Female & $56(52,8 / 27,7)$ & $70(72,9 / 34,7)$ & \\
\hline \multicolumn{4}{|l|}{ Age at treatment } \\
\hline 1st quartile (18-42 years) & $28(26.4 / 13.9)$ & $25(26.0 / 12.4)$ & 0.952 \\
\hline 2 nd quartile ( $43-53$ years) & $25(23.6 / 12.4)$ & $28(29.2 / 13.9)$ & 0.369 \\
\hline 3rd quartile (54-64 years) & $30(28.3 / 14.6)$ & $20(20.8 / 9.9)$ & 0.220 \\
\hline 4th quartile (65-86 years) & $23(21.7 / 11.4)$ & $23(24.0 / 11.4)$ & 0.703 \\
\hline \multicolumn{4}{|l|}{ Intubation time, secured airway } \\
\hline $1 \mathrm{~min}$. & $57(53.8 / 28.2)$ & $64(66.7 / 31.7)$ & 0.063 \\
\hline $2 \mathrm{~min}$. & $27(25.5 / 13.4)$ & $14(14.6 / 6.9)$ & 0.055 \\
\hline $3+\min$. & $22(20.8 / 10.9)$ & $18(18.8 / 8.9)$ & 0.722 \\
\hline \multicolumn{4}{|l|}{ Duration of intubation time } \\
\hline 1st quartile (58-142 $\mathrm{min}$.) & $28(26.4 / 13.9)$ & $25(26.0 / 12.4)$ & 0.952 \\
\hline 2nd quartile (143-175 min.) & $26(24.5 / 12.9)$ & $23(24.0 / 11.4)$ & 0.925 \\
\hline 3rd quartile (176-220 min.) & $25(23.6 / 12.4)$ & $28(29.2 / 13.9)$ & 0.369 \\
\hline 4th quartile (221-442 $\mathrm{min}$.) & $27(25.5 / 13.4)$ & $20(20.8 / 9.9)$ & 0.437 \\
\hline \multicolumn{4}{|l|}{ Pain } \\
\hline Mouth non or light (VAS 0-2) & $102(96.2 / 50.5)$ & $91(94.8 / 45.1)$ & 0.622 \\
\hline Mouth medium - severe (VAS 3-8) & $4(3.8 / 2.0)$ & $5(5.2 / 2.5)$ & 0.622 \\
\hline \multicolumn{4}{|l|}{ Pain } \\
\hline Throat non or light (VAS 0-2) & $101(95.3 / 50.0)$ & $89(92.7 / 44.1)$ & 0.441 \\
\hline Throat medium - severe (VAS 3-8) & $5(4.7 / 2.5)$ & $7(7.3 / 3.5)$ & 0.441 \\
\hline \multicolumn{4}{|l|}{ Hoarseness } \\
\hline Non or light $(0-2)$ & $106(100.0 / 52.5)$ & $94(97.9 / 46.5)$ & 0.136 \\
\hline Medium - severe (3-8) & $0(0.0 / 0.0)$ & $2(2.1 / 1.0)$ & 0.136 \\
\hline \multicolumn{4}{|l|}{ BMI } \\
\hline Underweight $(<18.5)$ & $3(2.8 / 1.5)$ & $0(0.0 / 0.0)$ & 0.098 \\
\hline Normal weight (18.5-24.9) & $46(43.4 / 22.8)$ & $44(45.8 / 21.8)$ & 0.728 \\
\hline Overweight (25-29.9) & $46(43.4 / 22.8)$ & $33(34.4 / 16.3)$ & 0.191 \\
\hline Severe overweight $(\geq 30)$ & $11(10.4 / 5.5)$ & $18(18.8 / 8.9)$ & 0.091 \\
\hline \multicolumn{4}{|l|}{ Number of intubation attempt } \\
\hline Normal (1-2) & $101(95.3 / 50.0)$ & $94(97.9 / 46.5)$ & 0.308 \\
\hline Over normal $(\geq 3)$ & $3(2.8 / 1.5)$ & $2(2.1 / 1.0)$ & 0.734 \\
\hline \multicolumn{4}{|l|}{ Number of anaesthetist involved } \\
\hline 1 & $102(96.2 / 50.5)$ & $86(89.6 / 42.6)$ & 0.064 \\
\hline 2 & $2(1.9 / 1.0)$ & $10(10.4 / 5.0)$ & $0.011^{*}$ \\
\hline Under bite & $99(93.4 / 49.0)$ & $90(93.8 / 44.6)$ & 0.919 \\
\hline
\end{tabular}

Changes during procedure according to intubation method: four times, three changes to laryngoscope, and one to fiberoptic. And according to tube size (standard no. 7 female and no. 8 male) in nine cases, the tube was changed to one size smaller.

Table 3. Pain and sore throat.

\begin{tabular}{|c|c|c|c|c|c|c|c|c|c|c|}
\hline & \multicolumn{3}{|l|}{ Pain mouth } & \multicolumn{3}{|c|}{ Pain throat } & \multicolumn{4}{|c|}{ Hoarseness } \\
\hline & $\begin{array}{l}\text { Mouth } \\
\text { (VAS 0-2), } \\
\text { n }\end{array}$ & $\begin{array}{l}\text { Mouth medium } \\
\text { to severe (VAS } \\
\text { 3-8), n }\end{array}$ & $\mathbf{P}$ & $\begin{array}{l}\text { Throat } \\
\text { (VAS 0- } \\
\text { 2), n }\end{array}$ & $\begin{array}{l}\text { Throat medium } \\
\text { to severe (VAS 3- } \\
\text { 8), n }\end{array}$ & $\mathbf{P}$ & $\begin{array}{l}\text { Non } \\
\text { (VAS 0), } \\
\text { n }\end{array}$ & $\begin{array}{l}\text { Light } \\
\text { (VAS } \\
\text { 1-2), n }\end{array}$ & $\begin{array}{l}\text { medium to } \\
\text { severe } \\
\text { (VAS 3-8), } \\
\text { n }\end{array}$ & $\mathbf{P}$ \\
\hline \multicolumn{11}{|l|}{ Gender } \\
\hline Male $n=76$ & 72 & 4 & 0.666 & 71 & 5 & 0.766 & 56 & 19 & 1 & 0.637 \\
\hline Female $n=126$ & 121 & 5 & & 119 & 7 & & 87 & 38 & 1 & \\
\hline 1st quartile ( $18-42$ years) $n=53$ & 49 & 4 & 0.205 & 50 & 3 & 0.920 & 36 & 15 & 2 & 0.173 \\
\hline 2nd quartile ( $43-53$ years) $n=53$ & 51 & 2 & 0.780 & 46 & 7 & $0.009 * *$ & 39 & 14 & 0 & 0.441 \\
\hline 3rd quartile ( $54-64$ years) $n=50$ & 49 & 1 & 0.333 & 49 & 1 & 0.175 & 36 & 14 & 0 & 0.620 \\
\hline 4 th quartile ( $65-86$ years) $n=46$ & 44 & 2 & 0.968 & 45 & 1 & 0.220 & 32 & 14 & 0 & 0.912 \\
\hline \multicolumn{11}{|l|}{ Intubation time, secured airway } \\
\hline 1 (min.) $n=121$ & 115 & 6 & 0.673 & 115 & 6 & 0.472 & 84 & 36 & 1 & 0.730 \\
\hline 2 (min.) $n=41$ & 40 & 1 & 0.484 & 40 & 1 & 0.289 & 30 & 11 & 0 & 0.551 \\
\hline \multicolumn{11}{|l|}{ Duration of intubation, time } \\
\hline 1st quartile (58-142 $\mathrm{min})$. & 52 & 1 & 0.292 & 49 & 4 & 0.566 & 38 & 14 & 1 & 0.886 \\
\hline 2nd quartile (143-175 min.) & 47 & 2 & 0.884 & 49 & 0 & $0.044^{*}$ & 35 & 14 & 0 & 0.688 \\
\hline 3rd quartile (176-220 min.) & 50 & 3 & 0.621 & 50 & 3 & 0.920 & 39 & 14 & 0 & 0.441 \\
\hline
\end{tabular}




\begin{tabular}{|c|c|c|c|c|c|c|c|c|c|c|}
\hline & \multicolumn{3}{|l|}{ Pain mouth } & \multicolumn{3}{|c|}{ Pain throat } & \multicolumn{4}{|c|}{ Hoarseness } \\
\hline & $\begin{array}{l}\text { Mouth } \\
\text { (VAS 0-2), } \\
\text { n }\end{array}$ & $\begin{array}{l}\text { Mouth medium } \\
\text { to severe (VAS } \\
\text { 3-8), n }\end{array}$ & $\mathbf{P}$ & $\begin{array}{l}\text { Throat } \\
\text { (VAS 0- } \\
\text { 2), n }\end{array}$ & $\begin{array}{l}\text { Throat medium } \\
\text { to severe (VAS 3- } \\
\text { 8), n }\end{array}$ & $\mathbf{P}$ & $\begin{array}{l}\text { Non } \\
\text { (VAS 0), } \\
\text { n }\end{array}$ & $\begin{array}{l}\text { Light } \\
\text { (VAS } \\
\text { 1-2), n }\end{array}$ & $\begin{array}{l}\text { medium to } \\
\text { severe } \\
\text { (VAS 3-8), } \\
\text { n }\end{array}$ & $\mathbf{P}$ \\
\hline $\begin{array}{l}\text { 4th quartile (221-442 } \mathrm{min} .) \\
\text { BMI }\end{array}$ & 44 & 3 & 0.466 & 42 & 5 & 0.121 & 31 & 15 & 1 & 0.289 \\
\hline Underweight $(<18.5) n=3$ & 3 & 0 & 0.707 & 3 & 0 & 0.662 & 2 & 1 & 0 & 0.943 \\
\hline Overweight (25-29.9) $n=79$ & 77 & 2 & 0.289 & 76 & 3 & 0.303 & 55 & 24 & 0 & 0.861 \\
\hline $\begin{array}{l}\text { Severe overweight }(\geq 30) n=29 \\
\text { Unknown, } n=1 \\
\text { Number of intubation attempts }\end{array}$ & 26 & 3 & 0.098 & 28 & 1 & 0.541 & 24 & 5 & 0 & 0.122 \\
\hline Normal (1-2) $n=195$ & 187 & 8 & 0.201 & 183 & 12 & 0.500 & 138 & 55 & 2 & 0.893 \\
\hline $\begin{array}{l}\text { Over normal }(\geq 3) n=5 \\
\text { Unknown, } n=2 \\
\text { Number of anaesthetist involved }\end{array}$ & 4 & 1 & 0.089 & 5 & 0 & 0.570 & 3 & 2 & 0 & 0.704 \\
\hline One nurse, $n=188$ & 181 & 7 & 0.065 & 177 & 11 & 0.844 & 134 & 53 & 1 & 0.164 \\
\hline $\begin{array}{l}\text { Two nurses, } n=12 \\
\text { Unknown, } n=2\end{array}$ & 10 & 2 & $0.035^{*}$ & 11 & 1 & 0.718 & 7 & 4 & 1 & 0.065 \\
\hline Underbite function (yes) $n=189$ & 181 & 8 & 0.560 & 178 & 11 & 0.783 & 132 & 55 & 2 & 0.262 \\
\hline
\end{tabular}

*significant, $\mathrm{p}<0.05$.

** highly significant, $\mathrm{p}<0.01$.

Table 4. Regression analysis of predictors for intubation with the oral fiberoptic bronchoscope-guided device versus the oral Macintosh laryngoscope-guided device.

\begin{tabular}{lll}
\hline & $\mathbf{9 5 \%}$ confidence interval & significance level, P \\
\hline Male & $0.07-0.35$ & $0.003^{*}$ \\
Patient safety & & \\
№ anaesthetist involved, one & $-1.18-0.17$ & 0.143 \\
№ anaesthetist involved, two & $-1.56-0.11$ & $0.024^{*}$ \\
BMI & & 0.122 \\
Underweight & $-0.12-0.99$ & 0.173 \\
Severe overweight & $-0.37-0.06$ & 0.053 \\
Patient comfort & & \\
Pain in the Throat/Neck & $-0.43-0.00$ & \\
\hline
\end{tabular}

*significant $<0.05$.

\section{Results}

Two hundred and nine patients were found eligible and included in the study, immediately before, during, or at the surgical procedure seven patients $(3.3 \%)$ were excluded due to the exclusion criterion (an acute situation, the need for changing the intubation procedure, and a few patients had not been fasting according to the guidelines). The final study consisted of two hundred and two patients scheduled for elective neurosurgery comprising cerebral tumour surgery; herniated discs in the spine; or cerebral external valve surgery. All surgical procedures required general anaesthesia using intratracheal intubation. The mean age was 52 years, between 20 and 86 years, and $62.4 \%$ of the patients were women. Table 1 contains detailed information on the baseline characteristics of the study cohort. Briefly, 106 (52.5\%) patients were allocated to the oral fiberoptic bronchoscope-guided intubation group (FI) and $96(47.5 \%)$ to the oral Macintosh laryngoscope intubation group (LI). More women $(n=126)$ than men $(n=76)$ were included in the study; almost twice as many men were in the FI group $(n=50)$ than included in the LI group $(n=26)$. This unequal distribution also occurred for the women, however, to a lesser degree and conversely in the two groups FI $(n=56)$ and LI $(n=70)$. Intubations until secured airway involved two nurse anaesthetists in $7.1 \%$ of the female cases and $4.0 \%$ of the male cases. Percentages of obesity with BMI of $\geq 25$, were less pronounced among women $n=61$ (48.4\%), compared to men $n=47(61.8 \%)$, in total over half of the participants were obese $(53.5 \%)$.

As illustrated in Table 2, differences in the patient comfort between the FI and LI group were minor. Corresponding to, medium to severe (VAS 3-8) pain in the mouth, as well as hoarseness as not existent or light (VAS 0-2), and medium to severe (VAS 3-8) hoarseness. Solely medium to severe pain in the throat (VAS $3-8$ ), stood out with a $\mathrm{P}=0.053$ predicting a weak association of a difference between groups, pointing at less pain in the FI group (Table 4). According to patient safety, the distribution of the time it took to secure a safety airway was, in most cases, between 1-2 minutes, in the LI group it took $\geq 3$ minutes in $18.8 \%$ of the cases compared to $20.8 \%$ in the FI group (Table 2), this difference was not statistically significant. In the category of intubation time 3 minutes or more, two patients experienced medium to severe pain in the mouth, and five experienced medium to severe pain in the throat (Table 3). The number of nurse anaesthetists involved differed significantly within groups 
with $\mathrm{P}=0.011$; two nurse anaesthetists were involved in two of the FI cases in contrast to the similar need in 10 cases when using LI (Table 2). This difference between groups was still statistically significant in the logistic regression analysis, with $\mathrm{P}=0.024$ (Table 4). Generally, the distribution of men and women in the two groups differed most noticeably for the LI group, with $27.1 \%$ men and $72.9 \%$ women (Table 2 ).

Furthermore, differences according to BMI was found between groups. It was found that $2.8 \%$ of patients were underweight $(\mathrm{BMI}<18.5)$ in the FI group versus $0 \%$ in the LI group; according to overweight (BMI 25-29.9) the distribution of patients was $43.4 \%$ of the FI group versus $34.4 \%$ of the LI group, and severe overweight (BMI $\geq 30$ ) distributed with $10.4 \%$ of the FI group and $18.8 \%$ within LI group (Table 2). Nevertheless, these differences were not statistically significant (Table 4). It was found that two patients with $\mathrm{BMI} \geq 25$ experienced medium to severe pain in the mouth, and three experienced medium to severe pain in the throat (Table 3).

\section{Discussion}

The present study provided information from a singleblinded, consecutively controlled clinical trial of men and women aged 20-86 years undergoing elective non-acute neurosurgical procedures in general anaesthesia. It is important to seek methods to prevent POST currently; numerous factors are known to contribute to the causation of POST and hoarseness [2, 17, 18].

The main finding in the present study when comparing the oral fiberoptic bronchoscopy-guided intubation device to the oral Macintosh laryngoscope: address a statistically significant difference in patient safety; this was measured as the number of nurse anaesthetists involved until secured airway, $\mathrm{P}=0.024$ (Table 4); fewer nurse anaesthetists was engaged in the intubation procedure when using the fiberoptic bronchoscopyguided intubation device. Prior research reporting on the number of anaesthetists engaged in the intubation procedure in studies of the fiberoptic bronchoscopy-guided intubation device, the oral Macintosh laryngoscope singularly, or in a comparative study was not found. Why the current finding somewhat needs to speak for itself. We conjecture the finding may be pointing towards better manageability and visibility of the larynx when using the oral fiberoptic bronchoscopyguided intubation device. The visibility of the larynx may, in particular, be significant in the treatment of obese patients. In our study, the distribution of obese patients (BMI $\geq 30$ ) was different with a $\mathrm{P}=0.091$ with more obese patients in the control group (the oral Macintosh laryngoscope) leading to more complex intubations in this group (Table 2).

Also according to patient safety, there were a small but non-significant difference between the groups concerning the time measured on the tube insertion time (intubation time); we considered this finding mainly to be related to the nurse anaesthetists lesser experiences of using the fiberoptic bronchoscopy-guided intubation device. After all, the number of nurse anaesthetists involved during intubation using the fiberoptic bronchoscopy-guided intubation device and the oral Macintosh laryngoscope is considered a relevant topic of investigation in future studies.

Differences between groups according to the patient selfreported level of pain in the mouth and throat are worth mentioning $(\mathrm{P}=0.053)$ although not a statistically significant finding. Thus, the finding may somewhat be interpreted as pointing towards the use of the fiberoptic bronchoscopyguided intubation device may increase patient comfort when compared to the oral Macintosh laryngoscope (Table 4); the existing research in the field reports postoperative pain in the throat are considered to be comprehensive [1-3, 17, 18, 22, 23].

In the current study, hoarseness was found as a minor POST complication, finding in total 57 patients $(28.2 \%)$ reported some degree of postoperative hoarseness (VAS 1-2), and only one woman and one man reported medium to severe (VAS 3-8) postoperative hoarseness (Table 3). Few studies report postoperative hoarseness as a single measurement. Heidegger et al. [21] did compare fiberoptic nasotracheal intubation and laryngoscopic intubation in an RCT study, the researchers found no differences of postoperative hoarseness between groups. In contrast, two studies comparing intubations using the laryngeal mask airway versus tracheal intubation a significant difference of postoperative hoarseness were found [24, 25], the laryngeal mask airway is considered more gentle and minimising the risk of irritation of the laryngeal and vocal folds tissue. Consequently, Radu et al. found in theirs RCT study a difference of postoperative hoarseness of $15 \%$ versus $40 \%$ between groups [24], and Jaensson et al. found in theirs non-randomized, prospective and longitudinal study a difference of $33 \%$ versus $57 \%$ [25]. Both studies found more noticeable postoperative hoarseness in the group of tracheal intubated patients. Postoperative hoarseness can be considered a sparsely studied area since only a few studies with different designs and measurement methods has reported this postoperative complication.

In the current research, new knowledge on factors relevant to patient safety and comfort emerged. The study was prioritised and chosen based on the nurse anaesthetists' interest and concerns of patient safety and comfort within the department; the topic is of interest among nurse anaesthetists, also found in earlier studies investigating the patient comfort and safety during the peri anaesthesia [26, 27].

The strength of the study is considered to be the number of participants, and the procedure of blinding the patients, the researchers, and the data collection group of nurse anaesthetists conducting the postoperative interviews. Also, the homogeneity of the nurse anaesthetists level of experiences of using the two compared intubation devices is considered as a strength of the study. All participating nurse anaesthetists are deemed to have an equivalent level of experiences using the oral fiberoptic bronchoscope-guided intubation device, since they were all trained based on the same program and similar circumstances prior to the study period began. The majority of the nurse anaesthetist had several years of experiences using the oral Macintosh laryngoscope intubation device, and only a few had 
experiences of approx one year.

Our study also has some limitations. The design of the randomisation process using the mixed envelopes is the main reason for the uneven distribution in the two groups, regarding the number of patients and gender. It is argued by Jaensson et el [25], that POST and hoarseness were not found to be dependent on gender difference. We assume the gender differences between groups in the current study are of minor concern, since we used the same procedure of genderspecific tube sizes as Jaensson et al [25].

Further, possible additional postoperative analgesic consumption was not registered; this may have had a minor effect on the outcome of interest; all patients received the standard postoperative analgesic treatment. The indications for additional postoperative analgesics are headache or pain in the cicatrix.

\section{The Implication for Practice and Conclusion}

Patients intubated with the fiberoptic bronchoscopeguided intubation device differs according to patient safety, but also slightly in POST and hoarseness, when comparing to patients in the control group intubated with the oral Macintosh laryngoscope-guided intubation device.

The study has demonstrated that despite general anaesthesia, when using the fiberoptic bronchoscopy-guided intubation device method can increase patient safety, and cause patientexperienced pain in the throat to a lesser degree. Although POST and hoarseness may be considered as a minor problem, it is our point of view, that, by decreasing this problem. The nurse anaesthetist can contribute to increasing patients' postoperative comfort. The amount of research addressing the patient perspective of postoperative complications seem to be scarce; we were only able to find one older study on the topic [28]. Together with the findings in the present study, this indicates a need for further research focusing on the patient perspective of POST, patient satisfaction and patient comfort after general anaesthesia, in order to understand the patients' priorities and experiences of postoperative complications. The fiberoptic bronchoscopy-guided intubation device is considered a valuable tool in airway management during general anaesthesia $[3,5,21]$.

In conclusion, the findings point towards increased patient safety and comfort in the intervention group of the fiberoptic bronchoscopy-guided intubation device; thus, suggesting that there may be a need for an update and reconsiderations of the peri anaesthesia guidelines regarding the standard intubation device. The study adds new knowledge useful for an adaption of the guideline, further intervention studies and studies of patients perspectives, are needed.

\section{Acknowledgements}

Our greatest appreciation and thanks to the entire staff of nurse anaesthetists, and all the anesthesiologists at the department for selflessly contributing to the study. We also like to thank both Senior Nurse Lone Therkelsen and the managers of the Department of Neuroanesthesiology for providing appropriate circumstances for the project implementation and the reporting of the study.

\section{References}

[1] El-Boghdadly K, Bailey CR, Wiles MD. Postoperative sore throat: a systematic review. Anaesthesia. 2016; 71 (6): 706-17.

[2] Biro P, Seifert B, Pasch T. Complaints of sore throat after tracheal intubation: a prospective evaluation. European journal of anaesthesiology. 2005; 22 (4): 307-11.

[3] Tachibana N, Niiyama Y, Yamakage M. Less postoperative sore throat after nasotracheal intubation using a fiberoptic bronchoscope than using a Macintosh laryngoscope: A double-blind, randomized, controlled study. Journal of clinical anesthesia. 2017; 39: 113-7.

[4] Xu YJ, Wang SL, Ren Y, Zhu Y, Tan ZM. A smaller endotracheal tube combined with intravenous lidocaine decreases post-operative sore throat - a randomized controlled trial. Acta anaesthesiologica Scandinavica. 2012; 56 (10): 1314-20.

[5] Aqil M, Khan MU, Mansoor S, Mansoor S, Khokhar RS, Narejo AS. Incidence and severity of postoperative sore throat: a randomized comparison of Glidescope with Macintosh laryngoscope. BMC anesthesiology. 2017; 17 (1): 127.

[6] Chang JE, Kim H, Han SH, Lee JM, Ji S, Hwang JY. Effect of Endotracheal Tube Cuff Shape on Postoperative Sore Throat After Endotracheal Intubation. Anesthesia and analgesia. 2017; 125 (4): 1240-5.

[7] Seo JH, Cho CW, Hong DM, Jeon Y, Bahk JH. The effects of thermal softening of double-lumen endobronchial tubes on postoperative sore throat, hoarseness and vocal cord injuries: a prospective double-blind randomized trial. British journal of anaesthesia. 2016; 116 (2): 282-8.

[8] Tanaka Y, Nakayama T, Nishimori M, Tsujimura Y, Kawaguchi M, Sato Y. Lidocaine for preventing postoperative sore throat. The Cochrane database of systematic reviews. 2015 (7): Cd004081.

[9] Park JH, Lee YC, Lee J, Kim H, Kim HC. The influence of high-dose intraoperative remifentanil on postoperative sore throat: a prospective randomized study: A CONSORT compliant article. Medicine. 2018; 97 (50): e13510.

[10] Kuriyama A, Maeda H, Sun R, Aga M. Topical application of corticosteroids to tracheal tubes to prevent postoperative sore throat in adults undergoing tracheal intubation: a systematic review and meta-analysis. Anaesthesia. 2018; 73 (12): 154656.

[11] Yang HL, Liu FC, Tsai SC, Tsay PK, Lin HT, Liu HE. Ketorolac Tromethamine Spray Prevents PostendotrachealIntubation-Induced Sore Throat after General Anesthesia. BioMed research international. 2016; 2016: 4582439.

[12] Kuriyama A, Aga M, Maeda H. Topical benzydamine hydrochloride for prevention of postoperative sore throat in adults undergoing tracheal intubation for elective surgery: a systematic review and meta-analysis. Anaesthesia. 2018; 73 (7): 889-900. 
[13] Zhao X, Cao X, Li Q. Dexamethasone for the prevention of postoperative sore throat: a systematic review and metaanalysis. Journal of clinical anesthesia. 2015; 27 (1): 45-50.

[14] Honarmand A, Safavi M, Safaei Arani A, Shokrani O. The efficacy of different doses of liquorice gargling for attenuating postoperative sore throat and cough after tracheal intubation. European journal of anaesthesiology. 2016; 33 (8): 595-6.

[15] Mayhood J, Cress K. Effectiveness of ketamine gargle in reducing postoperative sore throat in patients undergoing airway instrumentation: a systematic review. JBI database of systematic reviews and implementation reports. 2015; 13 (9): 244-78.

[16] Maruyama K, Sakai H, Miyazawa $H$, Toda N, Iinuma $Y$, Mochizuki N, et al. Sore throat and hoarseness after total intravenous anaesthesia. British journal of anaesthesia. 2004; 92 (4): 541-3.

[17] Higgins PP, Chung F, Mezei G. Postoperative sore throat after ambulatory surgery. British journal of anaesthesia. 2002; 88 (4): 582-4.

[18] Chen KT, Tzeng JI, Lu CL, Liu KS, Chen YW, Hsu CS, et al. Risk factors associated with postoperative sore throat after tracheal intubation: an evaluation in the postanesthetic recovery room. Acta anaesthesiologica Taiwanica: official journal of the Taiwan Society of Anesthesiologists. 2004; 42 (1): 3-8.

[19] Collins SR, Blank RS. Fiberoptic intubation: an overview and update. Respiratory care. 2014; 59 (6): 865-78; discussion 7880 .

[20] World Medical Association Declaration of Helsinki: ethical principles for medical research involving human subjects. Jama. 2013; 310 (20): 2191-4.

[21] Heidegger T, Starzyk L, Villiger CR, Schumacher S, Studer R,
Peter B, et al. Fiberoptic intubation and laryngeal morbidity: a randomized controlled trial. Anesthesiology. 2007; 107 (4): 585-90.

[22] Cirilla DJ, 2nd, Ngo J, Vaisman V, Daly C, Ata A, Sandison $\mathrm{M}$, et al. Does the incidence of sore throat postoperatively increase with the use of a traditional intubation blade or the GlideScope? Journal of clinical anesthesia. 2015; 27 (8): 64651.

[23] Lee JY, Sim WS, Kim ES, Lee SM, Kim DK, Na YR, et al. Incidence and risk factors of postoperative sore throat after endotracheal intubation in Korean patients. The Journal of international medical research. 2017; 45 (2): 744-52.

[24] Radu AD, Miled F, Marret E, Vigneau A, Bonnet F. Pharyngolaryngeal discomfort after breast surgery: comparison between orotracheal intubation and laryngeal mask. Breast (Edinburgh, Scotland). 2008; 17 (4): 407-11.

[25] Jaensson M, Gupta A, Nilsson U. Gender differences in sore throat and hoarseness following endotracheal tube or laryngeal mask airway: a prospective study. BMC anesthesiology. 2014; 14: 56 .

[26] Stomberg MW, Sjostrom B, Haljamae H. Routine intraoperative assessment of pain and/or depth of anaesthesia by nurse anaesthetists in clinical practice. Journal of clinical nursing. 2001; 10 (4): 429-36.

[27] Aagaard K, Laursen BS, Rasmussen BS, Sorensen EE. Interaction Between Nurse Anesthetists and Patients in a Highly Technological Environment. Journal of perianesthesia nursing: official journal of the American Society of PeriAnesthesia Nurses. 2017; 32 (5): 453-63.

[28] Macario A, Weinger M, Carney S, Kim A. Which clinical anesthesia outcomes are important to avoid? The perspective of patients. Anesthesia and analgesia. 1999; 89 (3): 652-8. 